\title{
Correlation Between Facial Form And Tooth Forn In Indian Population
}

\author{
Maya Vinothini
}

\begin{abstract}
A study was performed to correlate the relationship between facial form and Maxillary central incisor tooth form among undergraduate dental students in our institution. Forty dental students comprising of 20 males and 20 females were randomly selected as study subjects. Two standardized photograph was taken for each individual ( Facial photograph, intraoral photograph). Outlines were traced using Adobe photoshop .The mean values were evaluated and results were tabulated. Results failed to prove the Leon William's theory. Key words: Leon William's theory, toothform, facial form
\end{abstract}

\section{Introduction:}

Fabrication of functionally and esthetically acceptable denture is one of the prime aim of prosthodontics. . Most patients seek prosthodontic treatment for restoration of esthetics . Anterior teeth is not subjected to any occlusal load like posteriors.Hence esthetics is given importance during anterior teeth selection (1) While selecting anterior tooth, three factors should be considered namely size, form and colour of the tooth. Outline form the anterior teeth can be determined using many methods namely Patient's profile, typal form theory, dentogenic concept. In this study correlation between facial form and tooth form has been done to compare the results with that Leon William's typal form theory (1914). According to Leon william's theory shape of the teeth should be inverse of the shape of the face(2). Studies were conducted to re-examine leon William's typal form theory and to find the degree of correspondence between facial form and tooth form in the population of Zenica, Bosnia and Herzegovina.This study is done among the undergraduate students in our institution in the Indian population

\section{Materials and Methods:}

In this study thirty undergraduate dental students with full complexion studying in our institution were randomly selected between the age group of 20-23 years including 15 males and 15 females Inclusion criteria:

Dentulous aged 20-23 years

Completely dentate arch with presence or absence of third molars

Natural maxillary anteriors in good alignment.

\section{Exclusion criteria:}

Extensive carious lesion, incisal wear, tooth fracture, gingival hyperplasia of the maxillary anterior tooth.

Previous orthognathic surgery

Congenital or surgical defects or any anomalies of the tooth.

Restoration of maxillary anterior teeth and complete or partial veneer crown.

The outline form of face and maxillary central incisor were determined using the standardized method. Face form have been obtained by tracing the outline between the zygomas and the from the hairline to the gnathion. Right maxillary central incisor tooth outline form was determined using an outline tracing made around the buccal surface of the teeth, which corresponds to mesial and distal contours, the incisal edge and the cervical margin. correlation of the tracings of the facial outline form and tooth form was done by comparing photographic tracings using visual method

Procedure:

Each subject was seated upright on a chair with occlusal plane parallel to the floor. Two standardized photographs were taken for each subject using Single Lens Reflect camera mounted on a tripod stand adjusted according to individual patient.

1) Facial photographs

2) Maxillary central incisors

For each photograph standardized distance(distance was $150 \mathrm{~cm}$ in facial photographs and for maxillary incisors it is $150 \mathrm{~cm}$.A full face photograph with the closed lip was obtained with lens positioned parallel to the subject's 
face. An intraoral photograph of the maxillary central incisor was obtained using cheek retractors, with lens parallel to the labial surface of the teeth. The images were transferred to the computer and edited using Adobe photoshop CS5. (3).The outline tracings were taken separately.(4)
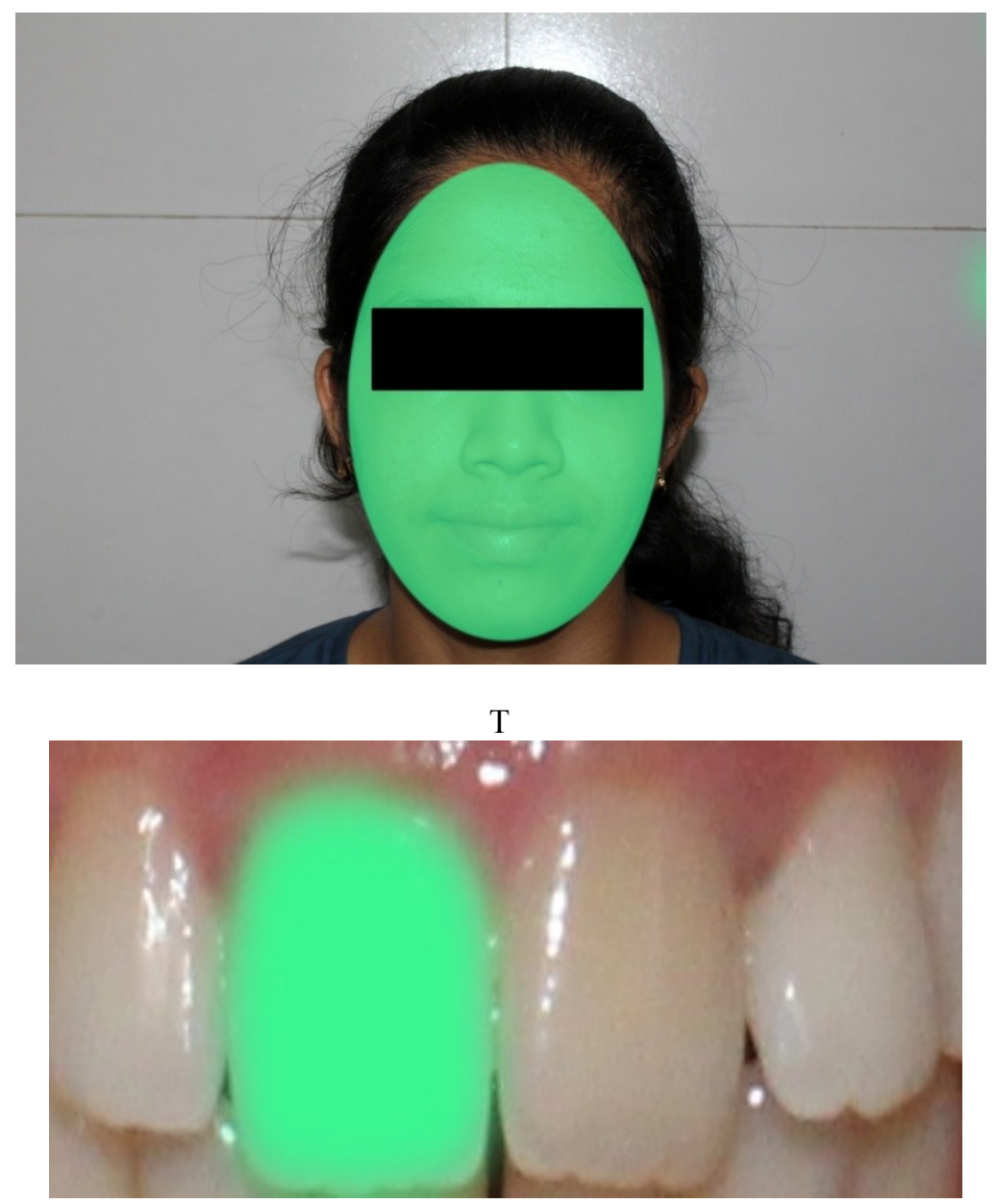

STATISTICS:

The mean values are taken, and the respective percentages were calculated and a stastics is formulated

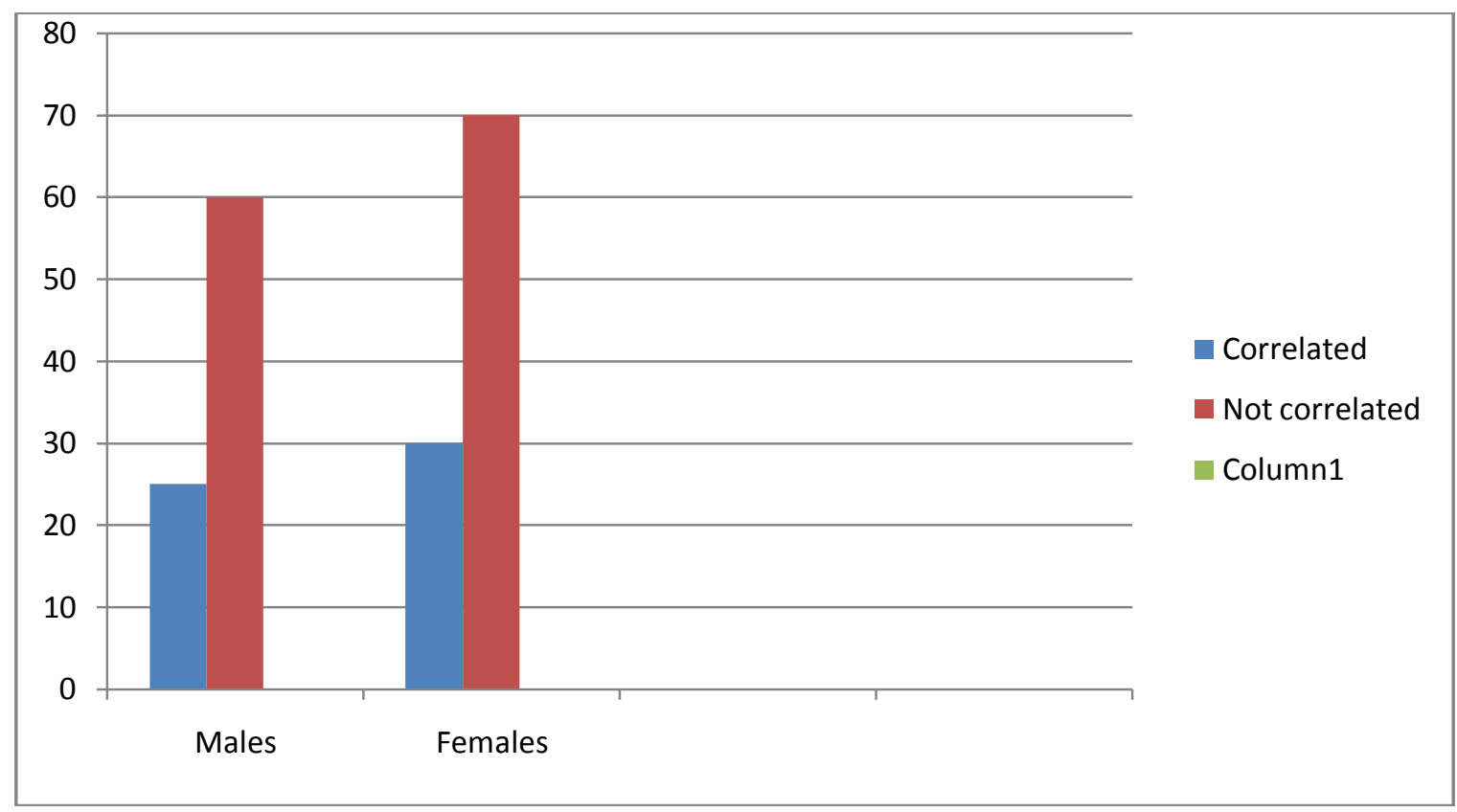




\section{Results:}

The results were obtained by correlating facial form and tooth form by photographic tracings using visual method. It was found that in $60 \%$ of the Males there was no correlation between facial form and tooth form according to leon William's theory and in only $25 \%$ of the individuals there was correlation between facial form and tooth form. Only in 30\% there was correlation between facial form and tooth form. And in 70\% there was no correlation between facial form and tooth form. This disproves the Leon William's typal form theory.

\section{References:}

[1] Textbook of Prosthodontics -Deepak Nallaswamy

[2] Bell RA(1978).The geometric theory of selection of artificial teeth:J Am Dent Assoc97,637-640

[3] Pavan kumar Journal of oral science, A clinical study to evaluate the correlation of facial form and tooth form in indiaVol.54,No.3,273-278,2012

[4] Varajo FM, Nogueria SS Russi Correlation between maxillary central incisor form and face form in 4 racial groups. 